\title{
Incommensurate standard map
}

\author{
Leonardo Ermann ${ }^{1,2}$ and Dima L. Shepelyansky ${ }^{3}$ \\ ${ }^{1}$ Departamento de Física Teórica, GIyA, Comisión Nacional de Energía Atómica, Avenida del Libertador 8250, \\ 1429 Buenos Aires, Argentina \\ ${ }^{2}$ Consejo Nacional de Investigaciones Científicas y Técnicas (CONICET), Buenos Aires, Godoy Cruz 2290, C1425FQB, CABA, Argentina \\ ${ }^{3}$ Laboratoire de Physique Théorique, IRSAMC, Université de Toulouse, CNRS, UPS, 31062 Toulouse, France
}

(Received 25 October 2018; published 24 January 2019)

\begin{abstract}
We introduce and study the extension of the Chirikov standard map when the kick potential has two and three incommensurate spatial harmonics. This system is called the incommensurate standard map. At small kick amplitudes, the dynamics is bounded by the isolating Kolmogorov-Arnold-Moser surfaces, whereas above a certain kick strength, it becomes unbounded and diffusive. The quantum evolution at small quantum kick amplitudes is somewhat similar to the case of the Aubru-André model studied in mathematics and experiments with cold atoms in a static incommensurate potential. We show that for the quantum map there is also a metalinsulator transition in space whereas in momentum we have localization similar to the case of two-dimensional Anderson localization. In the case of three incommensurate frequencies of the space potential, the quantum evolution is characterized by the Anderson transition similar to the three-dimensional case of the disordered potential. We discuss possible physical systems with such a map description including dynamics of comets and dark matter in planetary systems.
\end{abstract}

DOI: 10.1103/PhysRevE.99.012215

\section{INTRODUCTION}

The investigation of dynamical symplectic maps allows for understanding the fundamental deep properties of Hamiltonian dynamics. The map description originates from the construction of Poincaré sections of continuous dynamics invented by Poincaré [1]. The mathematical foundations for symplectic maps are described in Refs. [2,3]. Their physical properties and applications including numerical studies are given in Refs. [4,5]. The renormalization features of critical Kolmogorov-Arnold-Moser (KAM) invariant curves are analyzed in Ref. [6] with the transport properties through destroyed KAM curves investigated in Refs. [4,7].

The seminal example of a symplectic map is the Chirikov standard map [4],

$$
\bar{p}=p+K \sin x, \quad \bar{x}=x+\bar{p},
$$

where $p$ and $x$ are canonically conjugated variables of momentum $p$ and coordinate $x$ and bars mark the values of variables after a map iteration. It is argued that, for $K>$ $K_{c}=0.9716 \cdots$, the last KAM curve is destroyed and the dynamics is characterized by a global chaos and diffusion in momentum $\left\langle p^{2}\right\rangle=D t p$ with a diffusion rate $D$ where the time $t$ is measured in the number of map iterations and the diffusion coefficient is $D \approx K^{2} / 2$ at large $K$ values $[4,6,7]$.

The important feature of map (1) is its universality related to an equidistant spacing between resonance frequencies so that a variety of symplectic maps and periodically driven Hamiltonian systems can be locally described by the map (1) in a certain domain of the phase space. The Chirikov standard map finds applications for the description of various physical systems including plasma confinement in open mirror traps, microwave ionization of hydrogen atoms, comets, and dark matter dynamics in the solar system and behavior of cold atoms in kicked optical lattices (see, e.g., Refs. [4,8-11] and references therein).

The quantum version of map (1) is obtained by considering $p$ and $x$ as the Heisenberg operators with the commutation relation $[\hat{p}, \hat{x}]=-i \hbar$. The corresponding quantum evolution of the wave function $\psi(x)$ is described by the map $[12,13]$,

$$
\bar{\psi}=\exp (-i k \cos x) \exp \left(-i \hat{p}^{2} / 2 \hbar\right) \psi,
$$

where $\hat{p}=\hbar \hat{n}=-i \hbar \partial / \partial x, k=K / \hbar, T=\hbar, K=k T \quad(T$ can be also considered as a rescaled time period between kicks). Here, the wave function is defined in the domain $0 \leqslant x \leqslant 2 \pi$ corresponding to the quantum rotator case, or it can be considered on the whole interval $-\infty<x<\infty$ corresponding to motion of cold atoms in an optical lattice. The later case has been realized in experiments with cold atoms in a kicked optical lattice [14]. At $K>K_{c}$, the classical diffusion in $p$ becomes localized by the quantum interference effects with an exponential decay of probability over the momentum states $n=p / \hbar$,

$$
\left\langle\left|\psi_{n}\right|^{2}\right\rangle \propto \exp \left(-2\left|n-n_{0}\right| / \ell\right), \quad \ell \approx D / \hbar^{2},
$$

with the localization length $\ell$ and $n_{0}$ being an initial state $[12,15]$. This dynamical quantum localization is analogous to the Anderson localization in disordered solids as pointed out in Ref. [16]. However, the role of the spacial coordinate is played by momentum state level index $n$, and diffusion appears due to dynamical chaos in the classical limit and not due to disorder (see more details in Refs. [13,15,17]).

The important feature of the Chirikov standard map is its periodicity in spacial coordinate (of phase) $x$. The cases 
with several kick harmonics have been considered for various map extensions (see, e.g., Refs. $[4,5,12,18]$ ), but all of them had periodicity in $x$. The important example of the map with several harmonics is the generalized Kepler map which provides an approximate description of Halley's map dynamics in the solar system [19] (see also Ref. [11] for references and extensions). In this system, Jupiter gives an effective kick in the energy of Halley's comet when it passes through its perihelion. This kick function contains several sine harmonics of Jupiter's rotational phase since the comet perihelion distance is inside the Jovian orbit. However, other planets, especially Saturn, also give a kick change in comet energy. Since the frequencies of other planets are generally not commensurate with the Jovian rotation frequency, we have a situation where the kick function depends, at least, on two phases with incommensurate frequencies. Thus, it is important to analyze the incommensurate extensions of the Chirikov standard map.

The simplest model is the incommensurate standard ( $i$ standard) map which we investigate in this paper,

$$
\begin{aligned}
& \bar{p}=p+K_{1} \sin x+K_{2} \sin v x, \\
& \bar{x}=x+\bar{p},
\end{aligned}
$$

where $v$ is a generic irrational number and $K_{1}, K_{2}$ are amplitudes of two incommensurate kick harmonics. Here, the coordinate domain is $-\infty<x<\infty$ corresponding to dynamics of atoms in an incommensurate optical lattice. For $K_{1}=0$ or $K_{2}=0$, the model is reduced to the map (1). We consider here the case of the golden mean value of $v=(\sqrt{5}-1) / 2=0.618 \cdots$.

The incommensurate standard map (5) describes the dynamics of cold atoms in a kicked optical lattice with an incommensurate potential. The static incommensurate optical lattice can be created by laser beams with two incommensurate wavelengths. In fact, such an incommensurate optical lattice had been already realized in cold atoms experiments [20] where the evolution of atomic wave function can be approximately described [21] by the Aubry-André model on a discrete incommensurate integer lattice with the AubryAndré transition from localized to delocalized states [22]. At present, the investigation of interactions between atoms on such a lattice attracts a significant interest of the cold atom community (see, e.g., Ref. [23]).

Due to the above reasons, we think that the incommensurate standard map will capture new features of dynamical chaos with possible application to various physical systems. We also note that the quantum evolution of this map may have localization or delocalization properties with a certain similarity with the Anderson transition in disordered solids.

We note that the studies on incommensurate tori and related dynamics had been investigated by different groups (see, e.g., Refs. [24-26]) but the models considered there are not directly reducible to the incommensurate standard map and thus are different from the model considered here.

The paper is constructed as follows: Sec. II describes the properties of classical dynamics; quantum map evolution is analyzed in Sec. III, effective two-dimensional (2D) and three-dimensional (3D) features of quantum evolution are

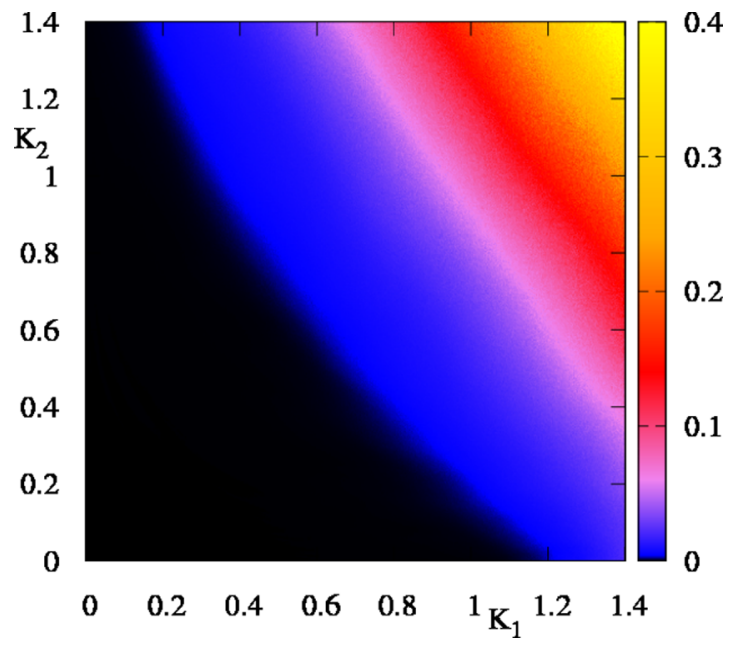

FIG. 1. Diffusion rate in momentum $D=\left\langle p^{2}\right\rangle / t$ vs $K_{1}$ and $K_{2}$. Data are averaged over 1000 trajectories with random initial conditions in the interval $x, p \in\left[0,10^{-6}\right)$ for the number of iterations $t=10^{4}$. The color bar shows the values of $D$, and the resolution in $\left(K_{1}, K_{2}\right)$ space is given by $\Delta K_{1}=\Delta K_{2}=0.003$.

considered in Sec. IV, and a discussion of the results is given in Sec. V.

\section{CLASSICAL MAP DYNAMICS}

To study the properties of classical dynamics of the map (5), we launch a bunch of trajectories in a vicinity of unstable fixed point $x=0, p=0$ and compute an effective diffusion coefficient $D=\left\langle p^{2}\right\rangle / t$ averaged over all initial trajectories. The dependence of $D$ on $K_{1}$ and $K_{2}$ is shown in Fig. 1 . These data show that there is a critical curve $K_{c 2}=f\left(K_{c 1}\right)$ below which the momentum oscillations are bounded and above which $p$ grows diffusely with time. At $K_{1}=K_{2}=K$, we obtain $K_{c}=K_{c 1}=K_{c 2} \approx 0.65$ (see below). Of course, the time $t$ used in Fig. 1 is not very long (due to many trajectories and many $K_{1}, K_{2}$ values) so that we obtain only an approximate position of the critical curve. Thus, for $K_{2}=0$ we know that $K_{c}=0.9716 \cdots$ [6], that is, a bit below than the blue domain with a finite diffusion rate $D$. This happens due to not very large $t=10^{4}$ value and a small diffusion near $K_{c}$ being $D \approx 0.3\left(K-K_{c}\right)^{3}$ for the map (1) [4,15].

To represent trajectories in the Poincare section, it is convenient to use variables $x_{1}=x(\bmod 2 \pi), x_{2}=v x(\bmod 2 \pi)$, and $p$. We show two sections for $K=K_{1}=K_{2}$ below the critical value at $K=0.2<K_{c}$ and above the critical value at $K=0.7>K_{c}$ (see Fig. 2 ). For $K=0.2$, there are smooth invariant KAM surfaces bounding $p$ variations, whereas, for $K=0.7$, there is a chaotic unbounded dynamics in momentum.

When the last KAM surface is destroyed, we have a diffusive growth of momentum as is shown in Fig. 3 for $K=$ $K_{1}=K_{2}=0.7>K_{c}$. For $K=K_{1}=K_{2}=0.2<K_{c}$, the numerically obtained values of $p$ remain bounded for all computational times.

From the time dependence of $\left\langle p^{2}\right\rangle$ on time $t$, we compute the diffusion rate $D$. The dependence of $D$ on $K=K_{1}=K_{2}$ is shown in Fig. 4. We find that this dependence is satisfactorily 

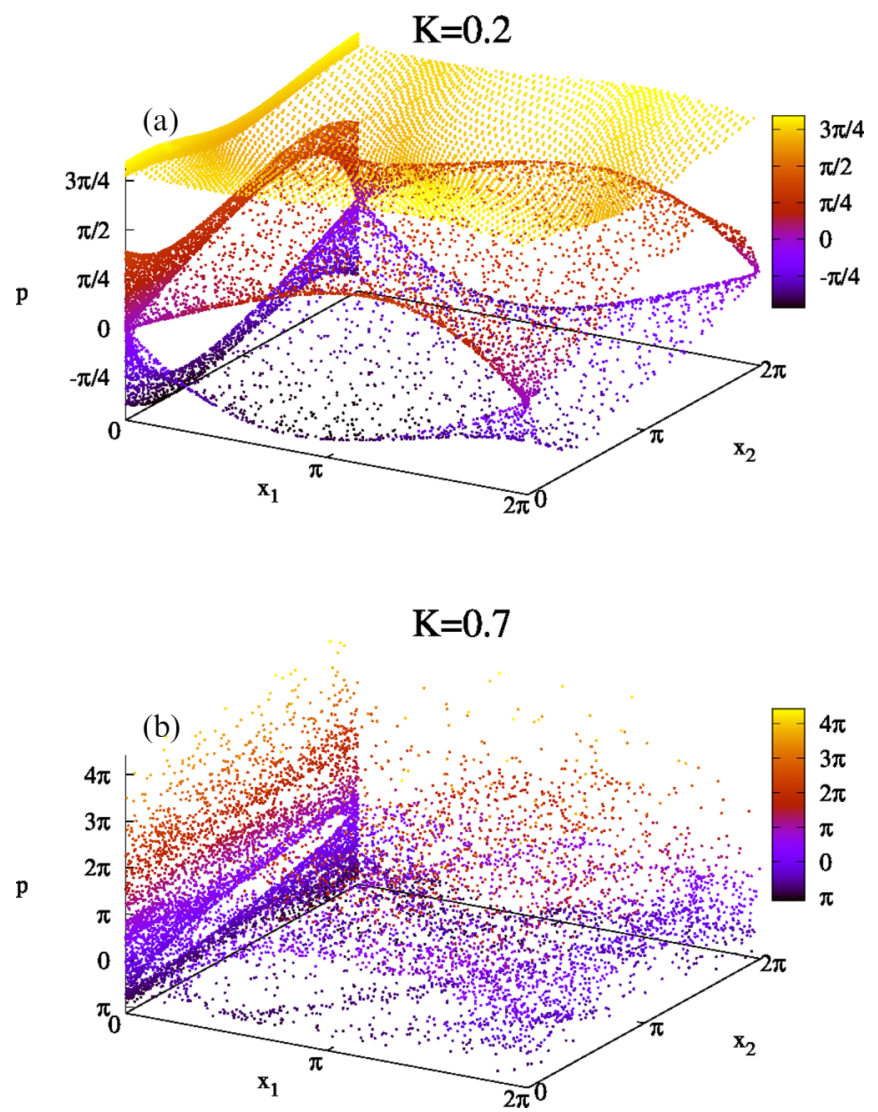

FIG. 2. The Poincaré section in the phase space $\left(x_{1}, x_{2}, p\right)$ for (a) $K=K_{1}=K_{2}=0.2$ and (b) $K=K_{1}=K_{2}=0.7$. (a) The data are obtained from two trajectories with $t=5000$ map iterations for the initial values of $p=x_{1}=0.01, x_{2}=0.01 \times v$ with $p=0.01$ and $p=\pi / \sqrt{2}$; (b) the initial phases $x_{1}, x_{2}$ are the same as in (a) and $p=0.01$. Color bars show $p$ values.

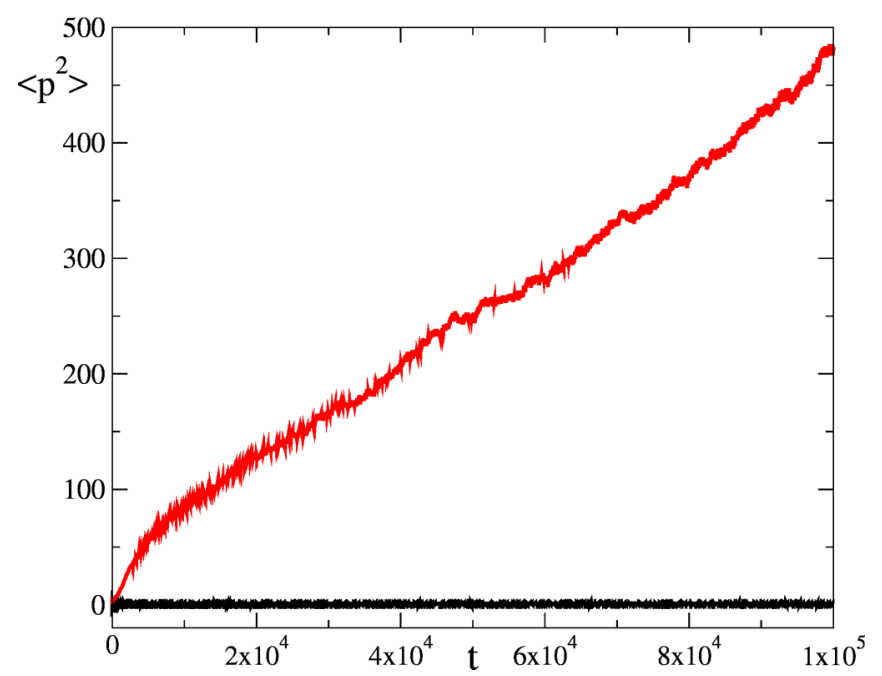

FIG. 3. The dependence of $\left\langle p^{2}\right\rangle$ on time $t$ for $K_{1}=K_{2}=0.2$ in the black (bottom) and $K_{1}=K_{2}=0.7$ in the red (gray) (top) solid curves. The average is performed over 1000 trajectories with random initial conditions in the range of $x, p \in\left[0,10^{-6}\right.$ ) (similar to initial conditions as in Fig. 1).

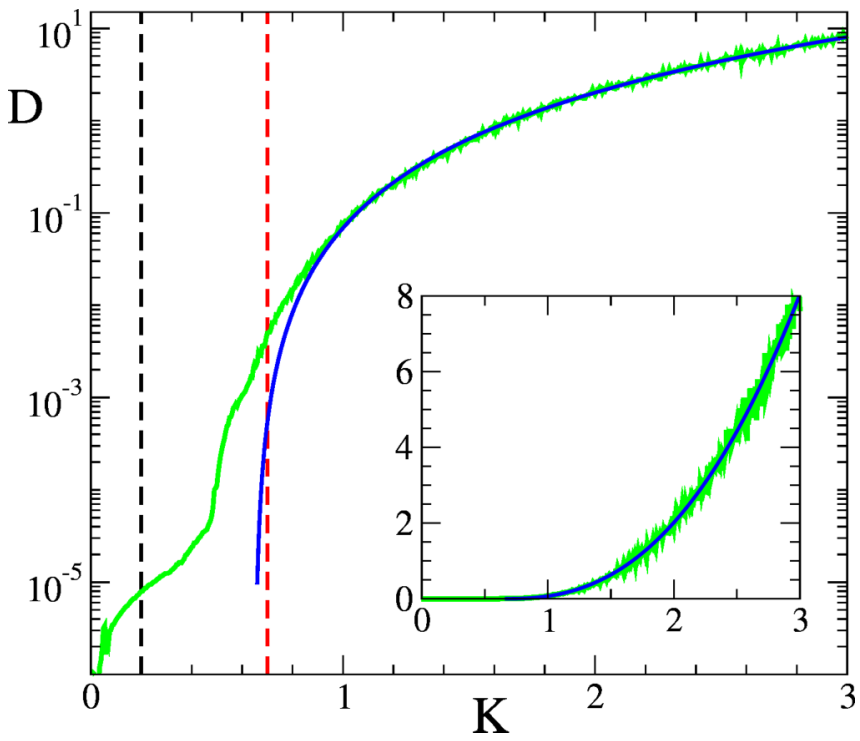

FIG. 4. Dependence of diffusion rate $D=\langle p\rangle / t$ on $K=K_{1}=$ $K_{2}$ shown by the green (gray) curve where the vertical black and red (gray) dashed lines mark values $K=K_{1}=K_{2}=0.2$ and $K=$ $K_{1}=K_{2}=0.7$, respectively. The fit dependence $D=D_{0}\left(K-K_{c}\right)^{\alpha}$ is shown by the blue (dark gray) curve with the fit values of $D_{0}=$ $0.95 \pm 0.04, k_{c}=0.65 \pm 0.02$, and $\alpha=2.5 \pm 0.03$. The inset plot shows the same curve for the linear scale on the $D$ axis. Data are obtained from 1000 trajectories and $t=10^{5}$ iterations.

described by the relation $D=D_{0}\left(K-K_{c}\right)^{\alpha}$ with $D_{0} \approx$ $0.95, K_{c} \approx 0.65$, and $\alpha \approx 2.5$. The value of the exponent $\alpha$ is close to the one for the Chirikov standard map with $\alpha \approx 3[4,7,15]$. Of course, the number of iterations $t=10^{5}$ is not very large, and due to that, we have only approximate values of the fit parameters $D_{0}, K_{c}, \alpha$. It is possible that the real value of $K_{c}$ is a bit smaller then its numerically obtained value of $K_{c}=0.65$ but a more exact determination of this value requires separate studies.

With the obtained global properties of the classical $i$ standard map, we go to the analysis of its quantum evolution in the next sections.

\section{QUANTUM MAP EVOLUTION}

The quantum evolution of the $i$-standard map is described by the following transformation of the wave function on one map period:

$$
\begin{aligned}
\bar{\psi}= & \exp \left\{-i\left[k_{1} \cos x+\left(k_{2} / v\right) \cos v x\right]\right\} \\
& \times \exp \left[-i \hat{p}^{2} / 2 \hbar\right] \psi,
\end{aligned}
$$

with $k_{1}=K_{1} / \hbar, k_{2}=K_{2} / \hbar$, and normalization condition $\int_{-\infty}^{\infty}|\psi(x)|^{2} d x=1$. Here, the wave function $\psi$ evolution is considered on infinite domain $-\infty<x<\infty$ corresponding to dynamics of cold atoms in kicked optical lattices; the first multiplier describes a kick from the optical lattice, and the second one gives a free propagation in empty space. The kick potential is $V(x)=k_{1} \cos x+\left(k_{2} / v\right) \cos v x$.

To perform numerical simulations of the quantum map (5), we approximate $v$ by its Fibonacci series with $v_{m}=$ $r_{m} / q_{m}=144 / 233$ considering the time evolution on a ring of 
size $\left[0,2 \pi q_{m}\right)$ with periodic boundary conditions. Here, $r_{m}=$ $f_{m-1}$ and $q_{m}=f_{m}$ are the $(m-1)$ th and the $m$ th Fibbonaci numbers given by $f_{m}=f_{m-1}+f_{m-2}$ with $f_{1}=f_{2}=1$, and therefore $\lim _{m \rightarrow \infty} v_{m}=v$. We use the Fibonacci series since they give the best rational approximants to the golden mean; they are usually used to investigate incommensurate effects in finite size systems (see, e.g., Refs. [5-7,21]). Then, one iteration of the map is obtained as

$$
\begin{aligned}
\bar{\psi}= & \hat{F}^{-1} \exp \left\{-i\left[k_{1} \cos \left(\frac{2 \pi x_{n}}{N_{0}}\right)+\frac{k_{2}}{v_{m}} \cos \left(\frac{2 \pi v_{m} x_{n}}{N_{0}}\right)\right]\right\} \hat{F} \\
& \times \exp \left[-i \frac{T}{2}\left(\frac{p_{n}}{q_{m}}\right)^{2}\right] \psi
\end{aligned}
$$

where $T=\hbar$ and the Hilbert space dimension is $N=N_{0} \times q_{m}$ with $q_{m}\left(r_{m}\right)$ periodic space cells of internal dimension $N_{0}$ for the $k_{1}\left(k_{2}\right)$ harmonic of $V(x)$. Here, positions and momentum have integer values of $x_{n}=n$ and $p_{n}=(n-\lfloor N / 2\rfloor)$ with $n=0, \ldots, N-1$; and $\hat{F}$ and $\hat{F}^{-1}$ are the operators of discrete Fourier transform from momentum to coordinate representation and back. We introduce these Fourier operators to indicate the way in which the numerical simulations are performed [of course, it is possible to present (6) in an operator form with operators for position and momentum]. For numerical simulations, we have chosen $N_{0}=3^{5}=243, r_{m}=144$, and $q_{m}=233$ with $\hbar=\frac{76 \pi}{3^{5}} \simeq 0.983$. We verified that the increase in rational approximants does not affect the results obtained.

We choose as an initial configuration a Gaussian wave packet centered at stable (or unstable) point of the kick potential $V(x)=k_{1} \cos x+\left(k_{2} / v\right) \cos v x$. On a discrete lattice of $x_{n}=n(0 \leqslant n<N)$, this distribution is

$\left.\psi\left(x_{n}\right)=A \exp \left\{-\left(x_{n}-X_{0}\right)^{2} /\left[2\left(N_{0} / 5\right)\right]^{2}\right)\right\}$ with $\langle x\rangle=X_{0}$ and $A$ as a normalization factor. The corresponding distribution, in momentum space $p_{n}$, is obtained by the discrete Fourier transform where, in this case, $\langle p\rangle=P_{0}=0$.

We define $P_{3, x}(t)$ and $P_{3, p}(t)$ as the probability to stay in three cells centered at initial $X_{0}$ and $P_{0}$ values, respectively,

$$
\begin{aligned}
& P_{3, x}(t)=\sum_{n=\left\lfloor X_{0}-\frac{3 N_{0}}{2}\right\rfloor}^{\left\lfloor X_{0}+\frac{3 N_{0}}{2}\right\rfloor}\left|\psi\left(x_{n}\right)\right|^{2}, \\
& P_{3, p}(t)=\sum_{n=\left\lfloor P_{0}-\frac{3 N_{0}}{2}\right\rfloor}^{\left\lfloor P_{0}+\frac{3 N_{0}}{2}\right\rfloor}\left|\psi\left(p_{n}\right)\right|^{2},
\end{aligned}
$$

where $\lfloor x\rfloor$ is the integer part of $x$.

The initial probability distributions placed in the vicinity of stable and unstable fix points of the kick potential $V(x)$ are shown in Fig. 5. We note that, for small $k_{1} \sim k_{2} \ll 1$, the map approximately describes a continuous time evolution in a static potential. In Ref. [21], it is shown that, in this case, the quantum evolution is approximately reduced to the AubryAndré model on a discrete lattice with the eigenstates of the stationary Schrödinger equation,

$$
\lambda \cos (\hbar n+\beta) \phi_{n}+\phi_{n+1}+\phi_{n-1}=E \phi_{n} .
$$

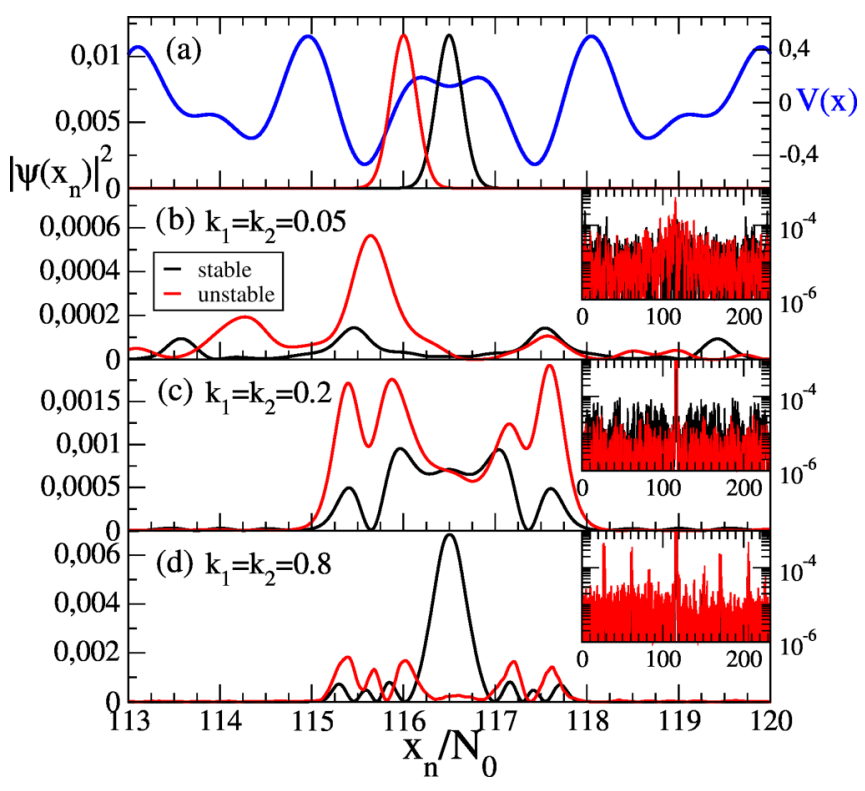

FIG. 5. Probability distribution in a coordinate. Panel (a) shows the probability distribution in a coordinate for the initial state given by a Gaussian centered at stable (black curves) and unstable [red (gray) curves] conditions. The blue (dark gray) curves show the potential energy $V(x)$ for $k_{1}=k_{2}=0.2$ where the scale is shown on the right side. Panels (b)-(d) (from top to bottom), respectively, show the distributions for $k_{1}=k_{2}=0.05, k_{1}=k_{2}=0.2$, and $k_{1}=$ $k_{2}=0.8$ after $t=10^{4}$ iterations with black and red curves showing the cases of stable and unstable initial conditions. In each panel, the top-right insets show the same distribution on the logarithmic scale and for the whole Hilbert space. In all cases, $T=76 \pi / 3^{5} \approx 0.983$.

Here, $\lambda$ is an effective dimensional energy of the quasiperiodic potential and the hopping amplitude being unity; here $\beta$ is a real number related to quasimomentum [21]. A metalinsulator transition (MIT) takes place from localized states at $\lambda>2$ to delocalized eigenstates at $\lambda<2$ [22]. A review of the properties of the Aubry-André model can be found in Ref. [27], and the mathematical proof of the MIT is given in Ref. [28]. An estimate obtained in Ref. [21] shows that $\lambda \propto k_{2} \exp \left(-C_{1} / k_{1}{ }^{C_{2}}+2 C_{3} \sqrt{k_{1}}\right)$ for an irrational $v \sim$ 1 with $C_{1}, C_{2}, C_{3}$ being numerical constant orders of unity. This approximate reduction of the Schrödinger equation in a continuous quasiperiodic potential $V(x)$ to the discrete lattice Aubry-André model have been used in experiments with cold atoms where the MIT was found at $\lambda=2[20,23]$.

The signs of the MIT are visible in Fig. 5 with a delocalization of probability in space at $k_{1}=k_{2}=0.05$ and localization at $k_{1}=k_{2}=0.2 ; 0.8$. At the same time, the results of Fig. 6 show that the probability distribution in momentum remains exponentially localized for the above the $k_{1}, k_{2}$ values.

The time evolution of probabilities of stay in a vicinity of initial cell $P_{3, x}$ and $P_{3, p}$ are shown in Figs. 7 and 8. We see that, at $k_{1}=k_{2}=0.05$, only a small fraction of probability (about 10\%) remains in a vicinity of initial space cell whereas in contrast it is rather large for $k_{1}=k_{2}=0.2 ; 0.8$. In contrast for the distribution in momentum about $99 \%$ remains in a vicinity of the initial cell for $k_{1}=k_{2}=0.05 ; 0.2$ and $65 \%$ for $k_{1}=k_{2}=0.8$. Thus, these data confirm localization in momentum and the MIT transition is space similar to the MIT 


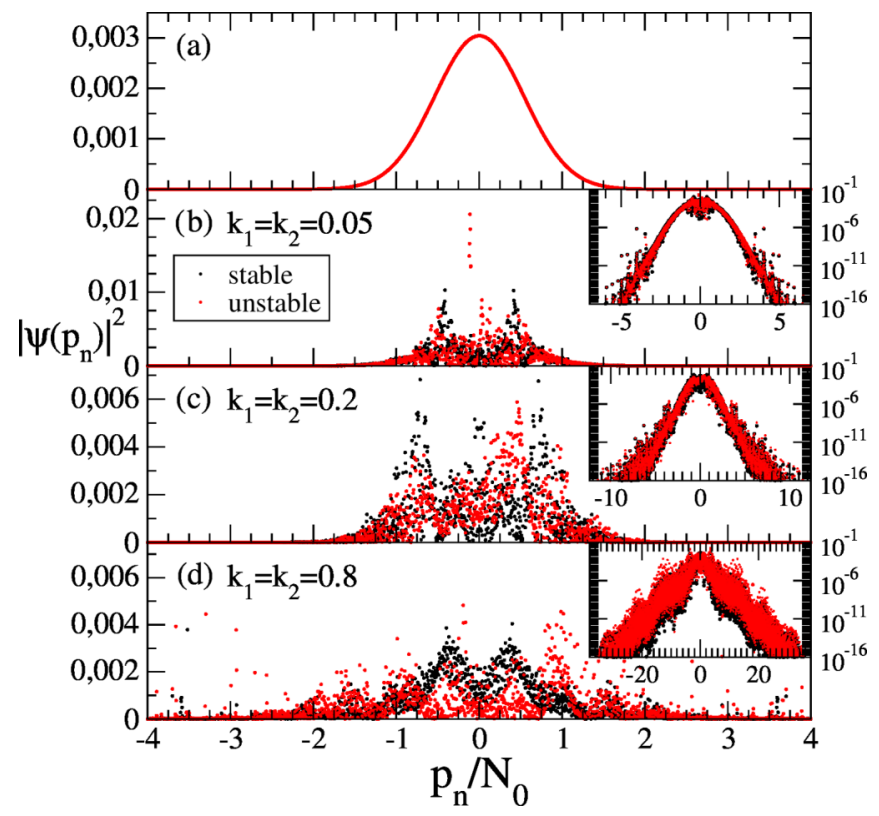

FIG. 6. Probability distribution in momentum for the same parameter values than Fig. 5. The top panel shows the probability distribution in momentum for the initial state given by a Gaussian centered at zero (the same for stable and unstable conditions). In the second, third, and fourth panels (from top to bottom) the distribution is shown for $k_{1}=k_{2}=0.05, k_{1}=k_{2}=0.2$, and $k_{1}=k_{2}=0.8$ after $t=10^{4}$ iterations where black and red dots illustrate the cases of stable and unstable conditions. In each panel, the top-right insets show the same distribution on the logarithmic scale and for the whole Hilbert space. In all cases, $T=76 \pi / 3^{5} \approx 0.983$.

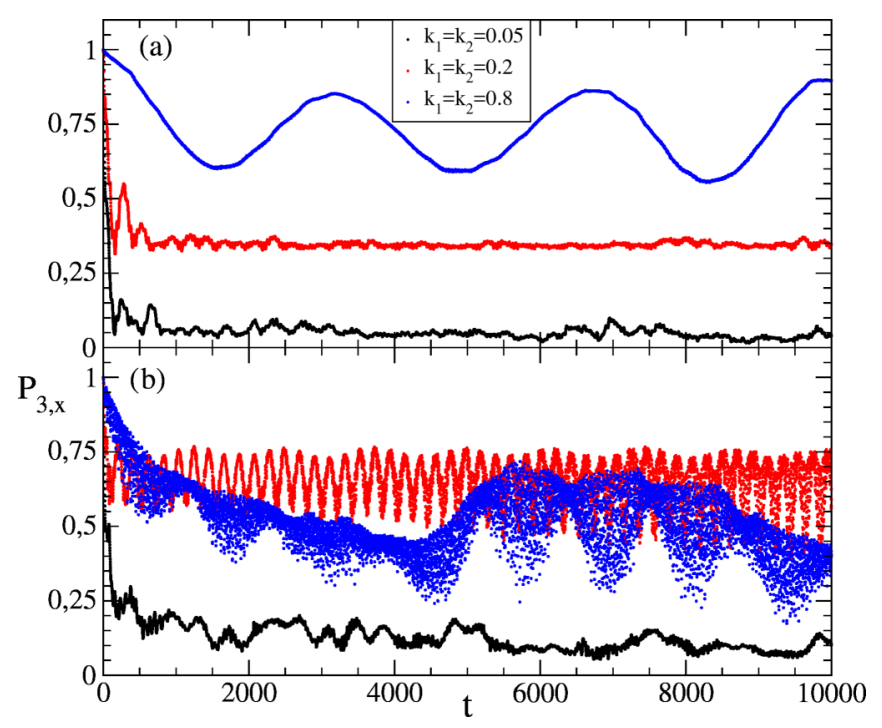

FIG. 7. Time evolution of the probability to stay in the first three space cells $P_{3, x}$ for the cases of Figs. 5 and 6 with $N_{0}=243$ and $q_{m}=233$. Top (a) and bottom (b) panels show the stable and unstable conditions, respectively, of Fig. 5 for $k_{1}=k_{2}=0.05$ (black circles), $k_{1}=k_{2}=0.2$ [red (gray) circles], and $k_{1}=k_{2}=0.8$ [blue (dark) gray circles] ordered from bottom to top in each panel [close to $t \sim 0$ for panel (b)].

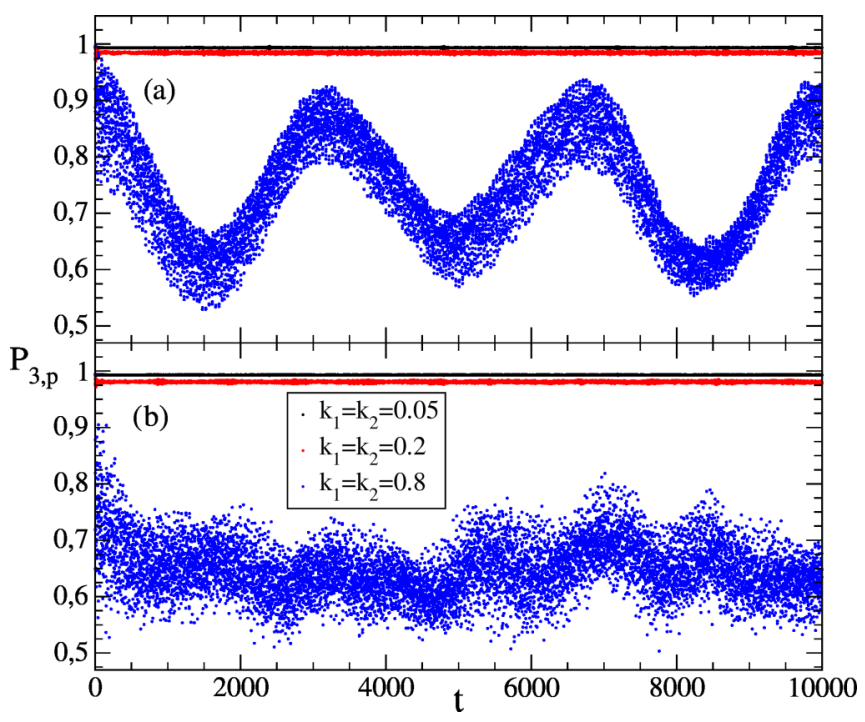

FIG. 8. Time evolution of the probability to stay in the first three momentum cells $P_{3, p}$ for the cases of Figs. 5 and 6 with $N_{0}=243$ and $q_{m}=233$. Top (a) and bottom (b) panels show the stable and unstable conditions, respectively, of Fig. 6 for $k_{1}=k_{2}=0.05$ (black circles), $k_{1}=k_{2}=0.2$ [red (gray) circles], and $k_{1}=k_{2}=0.8$ [blue (dark gray) circles] ordered from top to bottom in each panel.

discussed in Ref. [21] in the limit of the energy conservative system corresponding to the dynamics of our kicked model at small $k_{1}, k_{2}$ values.

The global dependence of probabilities $P_{3, x}(t)$ and $P_{3, p}(t)$ on kick amplitudes $k_{1}, k_{2}$ are shown in Figs. 9 and 10 for the initial packet centered in the vicinity of the stable or unstable point. For $P_{3, x}(t)$, there is a clear region on the $k_{1}, k_{2}$ plane where the probability $P_{3, x}(t)$ drops significantly corresponding to delocalization in space (see Fig. 9). In contrast, the probability $P_{3, p}(t)$ remains always rather high showing that the classical chaotic diffusion in momentum is localized by quantum interference effects.

In Fig. 11, we consider a case with a smaller value of $T=0.2$ so that the system becomes closer to the case of the stationary potential analyzed in Ref. [21]. These data are similar to the case at larger $T=0.983 \cdots$ with a domain of small probability $P_{3, x}(t)$ values at small $k_{1}, k_{2}$ indicated the
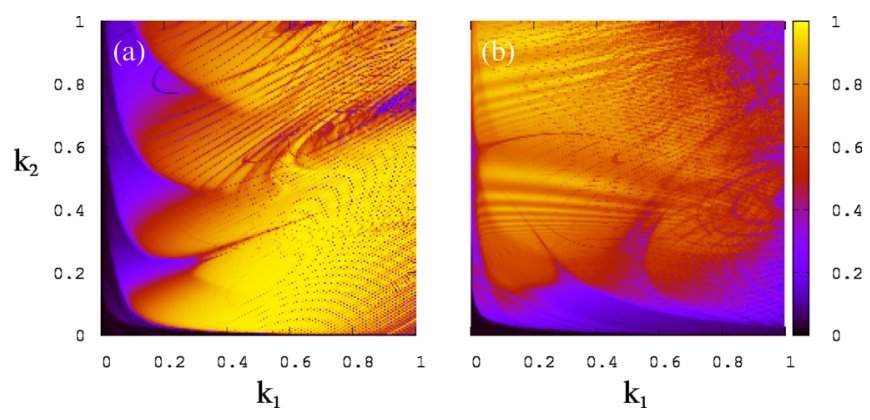

FIG. 9. Probability $P_{3, x}(t)$ averaged in time within interval $t \in$ $(9000,10000]$ is shown as a function of $k_{1}$ and $k_{2}$. Here, $T=$ $0.983 \cdots$, and the initial condition is centered at stable and unstable points of the potential in (a) and (b) panels, respectively. 

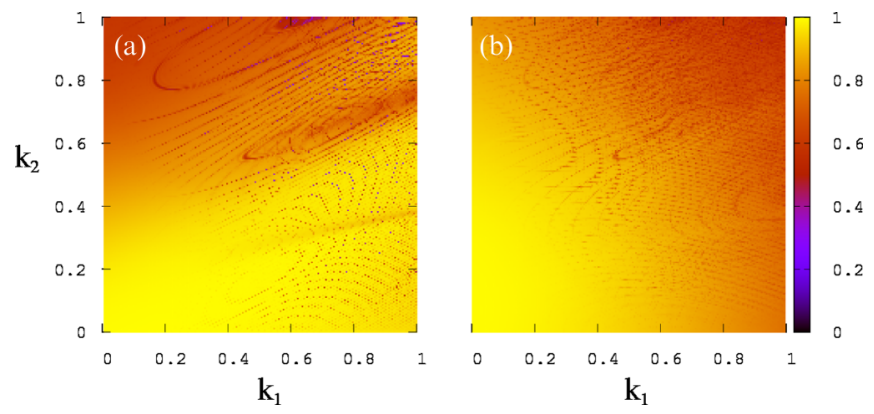

FIG. 10. Probability $P_{3, p}(t)$ in momentum averaged in time within interval $t \in(9000,10000]$ is shown as a function of $k_{1}$ and $k_{2}$. Here, $T=0.983 \cdots$, and the initial condition is centered at stable and unstable points of the potential in the left (a) and right (b) panels, respectively.

delocalization of probability in space. At the same time, the probability $P_{3, p}(t)$ in momentum remains always localized.

The obtained results show the presence of certain space delocalizations of the quantum incommensurate map at small kick amplitudes, whereas at large amplitudes the probability remains localized. The probability in momentum remains localized for all $k_{1}, k_{2}$ values at irrational $T / 2 \pi$ values.

At the same time, we note that the rigorous proof of probability delocalization in our model remains a mathematical challenge since the map of our system (or even its stationary version considered in Ref. [21]) on the Aubry-André model (9) works only approximately. Indeed, in our model it is possible to have excitation of high energy states (even if the numerical results indicate localization in momentum) that complicates the dynamics. We hope that the skillful mathematical tools developed in Ref. [28] will allow for obtaining mathematical results for the quantum incommensurate standard map.

Below, we consider the localization properties in momentum in more detail.

\section{TWO-DIMENSIONAL AND 3D MODELS OF THE $I$-STANDARD MAP}

To analyze the localization properties in momentum, we note that the kick with $k_{1}$ generates integer harmonics of
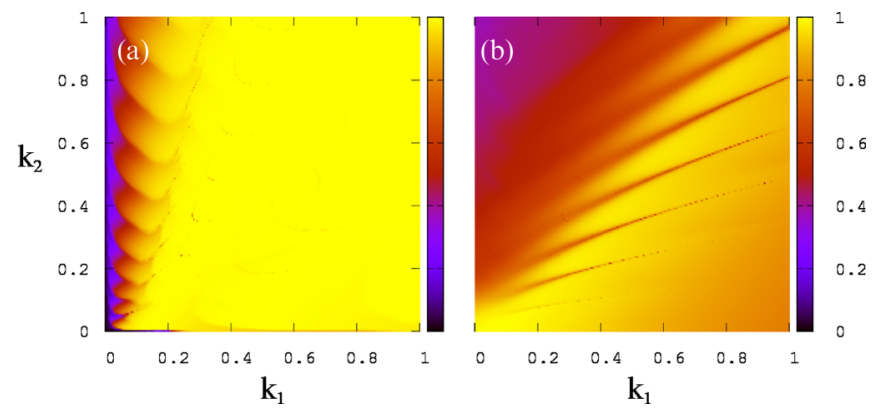

FIG. 11. Probability $P_{3, x}(t)$ in (a) space and $P_{3, p}(t)$ in (b) momentum averaged in time within interval $t \in(9000,10000]$ are shown as a function of $k_{1}$ and $k_{2}$. The initial condition in $x$ is centered at stable points of the potential and $T=0.2$. $\exp \left(-i j_{i} x\right)$ whereas the kick with $k_{2}$ generates only harmonics $\exp \left(-i j_{2} v x\right)$ with integer $j_{1}, j_{2}$ values. Due to the fact that the wave function contains only these two types of harmonics and the system evolution is described by

$$
\bar{\psi}=\hat{F}^{-1} e^{-i\left[k_{1} \cos x+k_{2} \cos v x\right]} \hat{F} e^{-i T\left(j_{1}+v j_{2}\right)^{2} / 2} \psi,
$$

where $\hat{F}$ is the 2D fast-Fourier transformation from momentum to space representation and $\hat{F}^{-1}$ gives a back transformation from space to momentum. The integers $j_{1}$ and $j_{2}$ number the correspondent harmonic numbers with the energy phase of free propagation between kicks being $\phi_{E}=T p^{2} / 2=T\left(j_{1}+\right.$ $\left.v j_{2}\right)^{2} / 2$ with $p=\left(j_{1}+v j_{2}\right)$. If we would have $\phi_{E}\left(j_{1}, j_{2}\right)$ taking random values for each $j_{1}, j_{2}$, then we would have a 2D kicked rotator with the Anderson type localization in 2D. In such a case, we would expect that the localization length $\lambda$ grows exponentially with the diffusion rate $\ln \lambda \sim$ $D \sim\left(k_{1}^{2}+k_{2}^{2}\right) / 2$ (see, e.g., Refs. [15,29,30]). However, the phases $\phi_{E}\left(j_{1}, j_{2}\right)$ are not random but incommensurate, and the appearance of 2D Anderson localization is not so obvious.

The fact that the model (10) corresponds to an effective 2D Anderson model is related to the fact that due to incommensurability of the kick potential in space we are getting two indices corresponding to two dimensions. The exact form and structure of the mapping on the $2 \mathrm{D}$ solid state lattice with effective disorder is discussed in detail for similar models in Refs. [15-17,29], and we do repeat this mapping here.

For the investigation of this expected 2D localization, we take $N_{2}=3^{7}$ harmonics $j_{1}$ and $j_{2}$ so that the total number of states becomes $N=N_{2}^{2}=4782969$. The results of the numerical fit show an exponential decay of probability with momentum $p$ as is shown in Fig. 12(a) for a few $k=k_{1}=k_{2}$ values. We fit this decay by an exponential dependence $|\psi(p)|^{2} \sim \exp (-|p| / \lambda)$ thus determining the localization length $\lambda$. The results presented in Fig. 12(b) show the expected exponential growth of localization length $\ln \lambda \sim$ $2.3 k$. The fact that $\ln \lambda$ is proportional to $k$ and not to expected $k^{2}$ can be attributed to the fact that we are still relatively close to the chaos border (see Fig. 4) and that the diffusion rate is small whereas the estimate $\ln \lambda \sim D \sim k^{2}$ assumes well developed chaotic regime with a relatively high $D$ [30].

There is no delocalization in $2 \mathrm{D}$, but in $3 \mathrm{D}$ there is the Anderson transition to delocalization [31] if a disorder is below a critical value or chaotic diffusion rate is higher a certain border (see, e.g., Ref. [30]). We argue that the 3D case can be realized in our model if we add kick with one more incommensurate potential $V_{3}(x)=k_{3} \cos v_{3} x$. Then, the wave function additional harmonics $\exp \left(-i j_{3} v_{3} x\right)$, and in analogy with (10), the time evolution is described by

$$
\begin{aligned}
\bar{\psi}= & \hat{F}^{-1} e^{-i\left[k_{1} \cos x+k_{2} \cos v x+k_{3} \cos \nu_{3} x\right]} \\
& \times \hat{F} e^{-i T\left(j_{1}+v j_{2}+\nu_{3} j_{3}\right)^{2} / 2} \psi,
\end{aligned}
$$

where momentum integer harmonics are $j_{1}, j_{2}, j_{3}=$ $1, \ldots, N_{3}$ and with the total dimension $N=N_{3}^{3}=529^{3}=$ 148035889 and $p=j_{1}+j_{2} / \theta+j_{3} / \theta^{2}$ with irrational $\theta=1.32471795724475 \cdots$ being the solution of equation $\theta^{3}-\theta-1=0$. Thus, $v=1 / \theta, v_{3}=1 / \theta^{2}$.

The results for time evolution of probability are presented in Fig. 13. They clearly show that, at $k=k_{1}=k_{2}=0.2$, there is exponential Anderson localization of probability over 

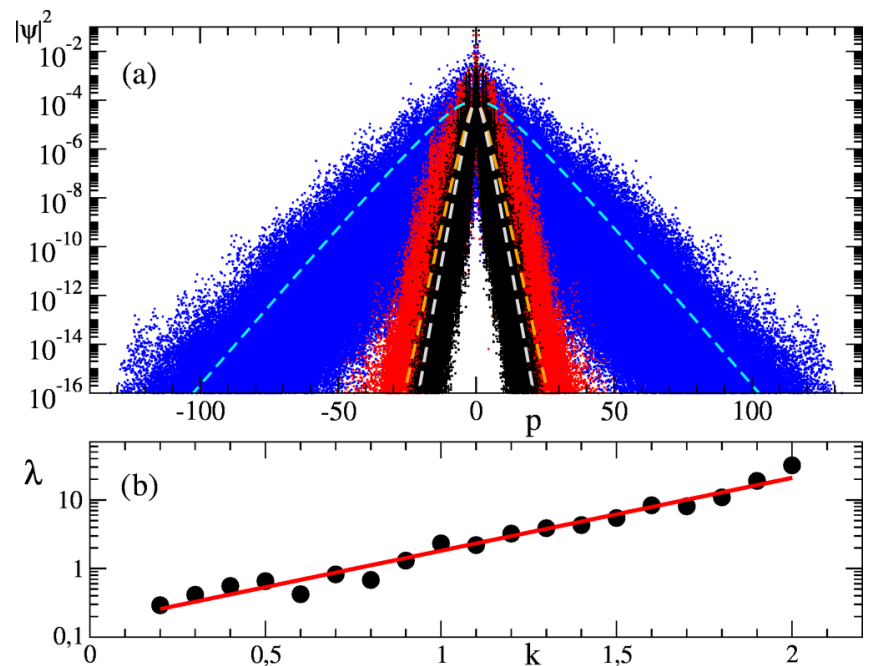

FIG. 12. (a) Probability distribution $\left|\psi\left(j_{1}, j_{2}\right)\right|^{2}$ in the model (10) as a function of $p=j_{1}+v j_{2}$ shown at time $t=10^{4}$. Here $T=2$ and $N_{2}=3^{7}=2187(N=4782969)$. The black (internal domain), red (gray) (middle domain), and blue (dark) (external domain) circles represent $k=k_{1}=k_{2}=0.5,0.7,1.2$, respectively. Numerical fits of $|\psi|^{2} \sim e^{-(p / \lambda)}$ with localization length values are shown by the dashes lines with $\lambda(k=0.5) \approx 0.656, \lambda(k=0.7) \approx 0.822$, and $\lambda(k=1.2) \approx 3.22$. Panel (b) shows the exponential dependence of fitted values of $\lambda$ as a function of $k$. The numerical fit is shown by the straight line with the exponential growth $\lambda \approx 0.16 \exp (2.44 k)$. The initial state is at $j_{1}=j_{2}=0$.

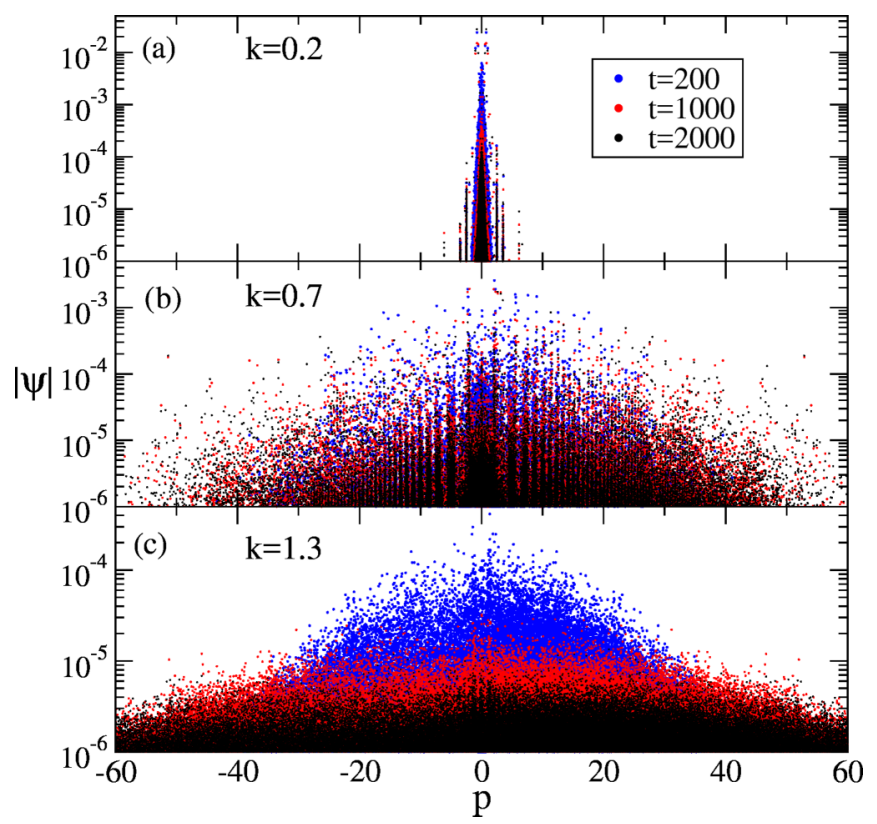

FIG. 13. Probability distribution $\left|\psi\left(j_{1}, j_{2}, j_{3}\right)\right|^{2}$ in the model (11) as a function of $p=j_{1}+j_{2} / \theta+j_{3} / \theta^{2}$ where $\theta$ is the solution of $\theta^{3}-\theta-1=0$. Here, $T=2$ and $N_{3}=529\left(N=N_{3}{ }^{3}=\right.$ 148035889 ) at evolution times $t=200,1000,2000$ with blue (top dark points), red (middle gray points), and black (bottom) points, respectively. Top (a), middle (b), and bottom (c) panels show the cases of $k_{1}=k_{2}=k_{3}=0.2, k_{1}=k_{2}=k_{3}=0.7$, and $k_{1}=k_{2}=$ $k_{3}=1.3$. The initial state is at $j_{1}=j_{2}=j_{3}=0$. The Anderson transition takes place at $k=k_{1}=k_{2} \approx 0.7$. momentum [31]. For $k=k_{1}=k_{2}=1.3$, there is spreading of probability in time over momentum states. The case at $k=k_{1}=k_{2}=0.7$ is close to a critical parameter value where the Anderson transition takes place. Thus, the MIT point is located in the range of $0.7 \leqslant k_{c}<1.3$. Additional studies should be performed to obtain the critical parameter more exactly, but the presented results definitely show that the transition takes place for the $k$ parameter in this range.

The results of this section show that in the model (10) the wave functions are always exponentially localized in the momentum space but their localization length grows exponentially with the kick amplitude whereas for the model (11) the localization takes place for relatively weak kick amplitude whereas for the amplitude being larger than a certain threshold a delocalization takes place in the momentum space being similar to the Anderson transition in 3D.

Due to the similarity with the 2D and 3D Anderson localizations we can say that there is also a certain similarity with the frequency modulated kicked rotator introduce in Ref. [32] and further investigated in other works (see, e.g., Refs. [29,33,34]). However, there are also certain differences since here we have incommensurability in space whereas in the models of Refs. [29,32] there is incommensurability in time. Also, in the 1D case, the presence of Aubry-Andre type transition clearly shows the difference between two models of incommensurability.

\section{DISCUSSION}

In our research, we determined the main properties of the incommensurate standard map for its classical dynamics and for its quantum evolution. In the classical case, the invariant KAM surfaces are destroyed above certain kick amplitudes, which gives us a critical curve on the plane of kick amplitudes $K_{1}, K_{2}$ (see Fig. 1). We find that above the critical curve at its vicinity the diffusion rate is characterized by a critical exponent $\alpha \approx 2.5$ which is not so far from the case of the Chirikov standard map.

The quantum evolution at small quantum kick amplitudes $k_{1}=K_{1} / \hbar, k_{2}=K_{2} / \hbar$ is similar to the Aubry-André type transition [22] as discussed in Ref. [21] and observed in cold atom experiments with a static incommensurate potential [20,23]. However, at larger values of $k_{1}, k_{2}$ the evolution remain localized both in space and momentum. We show that the localization in momentum is similar to the case of Anderson localization in 2D. Whereas significant progress has been reached with rigorous results for the Aubry-André model [28], we point that the mathematical prove of space and momentum localization for the quantum incommensurate standard map represents a high challenge for mathematicians.

The quantum evolution for the quantum $i$-standard map is always localized in momentum as in the case of 2D Anderson localization. However, for three kick harmonics, the situation becomes similar to the 3D Anderson localization with the MIT from localized to delocalized evolution as the kick amplitude is increased. We note that this behavior has similarities with the frequency modulated kicked rotator introduced in Ref. [32] which also demonstrates the Anderson localization in effective two and three dimensions [29] observed in the 
cold atoms experiments [33,34]. Thus, the kicked rotator with one additional modulation frequency in the time domain is similar to the case of 2D Anderson localization in agreement with predictions performed in 1983 [32]. The case of two additional modulation frequencies is similar to the case of $3 \mathrm{D}$ Anderson transition as discussed in Ref. [29]. We hope that the results presented here will allow for investigating the Anderson localization in 2D and 3D for the periodically kicked rotator with a kicked incommensurate potential discussed in this paper.

As was pointed in Sec. I, the incommensurate standard map naturally appears for a description of dynamics of dark matter or comets in the solar system and other planetary systems with two or more planets rotating around the central star. Recently, it has been shown that in the case of star and one rotating planet the quantum effects can play a significant role for escape of very light dark matter from the planetary system due to the Anderson localization of energy transitions [35]. The obtained results show that the presence of a second planet leads to the dynamics described by the incommensurate standard map with significant effects on the quantum localization of dark matter. Indeed, in Ref. [19], it is shown that the dy- namics of Halley's comet is well described by the generalized Kepler map with incommensurate kicks produced by Jupiter and Saturn which give the energy change being similar to the incommensurate standard map (incommensurability appears due to incommensurability of rotation periods of Jupiter and Saturn). The map derivation and dynamics description of dark matter in a system of star with one planet is described in Ref. [11]; in the presence of the second planet, there will be two incommensurate kick functions similar to those studied here.

Since the Chirikov standard map has many universal features and appears in the description of evolution of many very different physical systems, we argue that the incommensurate standard map will also find a broad field of applications.

\section{ACKNOWLEDGMENTS}

This work was supported, in part, by the Programme Investissements d'Avenir ANR-11-IDEX-0002-02, reference ANR-10-LABX-0037-NEXT (Project THETRACOM). This work was granted access to the HPC resources of CALMIP (Toulouse) under Allocation No. 2018-P0110.
[1] H. Poincaré, Sur le problème des trois corps et les équations de la dynamique, Acta Math. 13, 5 (1890).

[2] V. I. Arnold and A. Avez, Ergodic Problems of Classical Mechanics (Benjamin, Paris, 1968).

[3] I. P. Cornfeld, S. V. Fomin, and Y. G. Sinai, Ergodic Theory (Springer, New York, 1982).

[4] B. V. Chirikov, A universal instability of many-dimensional oscillator systems, Phys. Rep. 52, 263 (1979).

[5] A. J. Lichtenberg and M. A. Lieberman, Regular and Chaotic Dynamics (Springer, Berlin, 1992).

[6] R. S. MacKay, A renormalisation approach to invariant circles in area-preserving maps, Physica D 7, 283 (1983).

[7] J. D. Meiss, Symplectic maps, variational principles, and transport, Rev. Mod. Phys. 64, 795 (1992).

[8] B. Chirikov and D. Shepelyansky, Chirikov standard map, Scholarpedia 3, 3550 (2008).

[9] M. Raizen and D. A. Steck, Cold atom experiments in quantum chaos, Scholarpedia 6, 10468 (2011).

[10] D. Shepelyansky, Microwave ionization of hydrogen atoms, Scholarpedia 7, 9795 (2012).

[11] J. Lages, D. Shepelyansky, and I. I. Shevchenko, Kepler map, Scholarpedia 13, 33238 (2018).

[12] B. V. Chirikov, F. M. Izrailev, and D. L. Shepelyansky, Dynamical Stochasticity in Classical and Quantum Mechanics, edited by S. P. Novikov, Soviet Scientific Reviews, Section C: Mathematical Physics Reviews (Harwood Academic Publishers, Switzerland, 1981), Vol. 2, p. 209.

[13] B. V. Chirikov, F. M. Izrailev and D. L. Shepelyansky, Quantum chaos: localization vs. ergodicity, Physica D 33, 77 (1988).

[14] F. L. Moore, J. C. Robinson, C. F. Bharucha, B. Sundaram, and M. G. Raizen, Atom Optics Realization of the Quantum $\delta$-Kicked Rotor, Phys. Rev. Lett. 75, 4598 (1995).

[15] D. L. Shepelyansky, Localization of diffusive excitation in multi-level systems, Physica D 28, 103 (1987).
[16] S. Fishman, D. R. Grempel, and R. E. Prange, Chaos, Quantum Recurrences and Anderson Localization, Phys. Rev. Lett. 49, 509 (1982).

[17] S. Fishman, Anderson localization and quantum chaos maps, Scholarpedia 5, 9816 (2010).

[18] K. M. Frahm and D. L. Shepelyansky, Diffusion and localization for the Chirikov typical map, Phys. Rev. E 80, 016210 (2009).

[19] B. V. Chirikov and V. V. Vecheslavov, Chaotic dynamics of comet Halley, Astron. Astrophys. 221, 146 (1989).

[20] G. Roati, C. D'Errico, L. Fallani, M. Fattori, C. Fort, M. Zaccanti, G. Modugno, M. Modugno, and M. Inguscio, Anderson localization of a non-interacting Bose-Einstein condensate, Nature (London) 453, 895 (2008).

[21] M. Modugno, Exponential localization in one-dimensional quasi-periodic optical lattices, New J. Phys. 11, 033023 (2009).

[22] S. Aubry and G. André, Analyticity breaking and Anderson localization in incommensurate lattices, Ann. Isr. Phys. Soc. 3, 18 (1980).

[23] M. Schreiber, S. S. Hodgman, P. Bordia, H. P. Luschen, M. H. Fischer, R. Vosk, E. Altman, U. Schmeider, and I. Bloch, Observation of many-body localization of interacting fermions in a quasirandom optical lattice, Science 349, 842 (2015).

[24] F. Borgonovi and D. L. Shepelyansky, Breaking of analiticity in 2 coupled Frenkel-Kontorova chains, Europhys. Lett. 21, 413 (1993).

[25] À. Haro and R. de la Llave, A parameterization method for the computation of invariant tori and their whiskers in quasiperiodic maps: Numerical algorithms, Discrete and Continuous Dynamical Systems Series B 6, 1261 (2006).

[26] J.-L. Figueras and A. Haro, Different scenarios for hyperbolicity breakdown in quasiperiodic area preserving twist maps, Chaos 25, 123119 (2015). 
[27] J. B. Sokoloff, Unusual band structure, wave functions and electrical conductance in crystals with incommensurate periodic potentials, Phys. Rep. 126, 189 (1985).

[28] S. Jitomirskaya, Metal-insulator transition for the almost Mathieu operator, Ann. Math. 150, 1159 (1999).

[29] F. Borgonovi and D. L. Shepelyansky, Two interacting particles in an effective 2-3-d random potential, J. Phys. I (France) 6, 287 (1996)

[30] Y. Imry, Introduction to Mesoscopic Physics (Oxford University Press, New York, 2002).

[31] P. W. Anderson, Absence of diffusion in certain random lattices, Phys. Rev. 109, 1492 (1958).
[32] D. L. Shepelyansky, Some statistical properties of simple classically stochastic quantum systems, Physica D 8, 208 (1983).

[33] J. Chabe, G. Lemarie, R. Cremaud, D. Delande, P. Szriftgiser, and J. C. Garreau, Experimental Observation of the Anderson Metal-Insulator Transition with Atomic Matter Waves, Phys. Rev. Lett. 101, 255702 (2008).

[34] I. Manai, J.-F. Clement, R. Chicireanu, C. Hainaut, J. C. Garreau, P. Szriftgiser, and D. Delande, Experimental Observation of Two-Dimensional Anderson Localization with the Atomic Kicked Rotor, Phys. Rev. Lett. 115, 240603 (2015).

[35] D. L. Shepelyansky, Quantum chaos of dark matter in the Solar system, arXiv:1711.07815. 